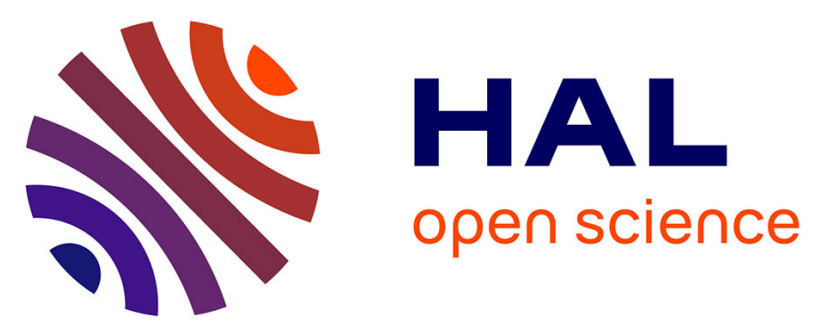

\title{
Croissance de la productivité et réallocations d'emplois au Maroc: la contribution des créations et disparitions d'entreprises
}

\author{
Richard Duhautois, Said El Hamine, Amin El Basri
}

\section{- To cite this version:}

Richard Duhautois, Said El Hamine, Amin El Basri. Croissance de la productivité et réallocations d'emplois au Maroc: la contribution des créations et disparitions d'entreprises. 2006. hal-00831539

\author{
HAL Id: hal-00831539 \\ https://hal.science/hal-00831539
}

Preprint submitted on 7 Jun 2013

HAL is a multi-disciplinary open access archive for the deposit and dissemination of scientific research documents, whether they are published or not. The documents may come from teaching and research institutions in France or abroad, or from public or private research centers.
L'archive ouverte pluridisciplinaire HAL, est destinée au dépôt et à la diffusion de documents scientifiques de niveau recherche, publiés ou non, émanant des établissements d'enseignement et de recherche français ou étrangers, des laboratoires publics ou privés. 


\title{
DOCUMENT DE TRAVAIL
}

\section{CROISSANCE DE LA PRODUCTIVITÉ}

\author{
ET RÉALLOCATIONS D'EMPLOIS
}

AU MAROC :

\section{LA CONTRIBUTION DES CRÉATIONS}

\section{ET DISPARITIONS D'ENTREPRISES}

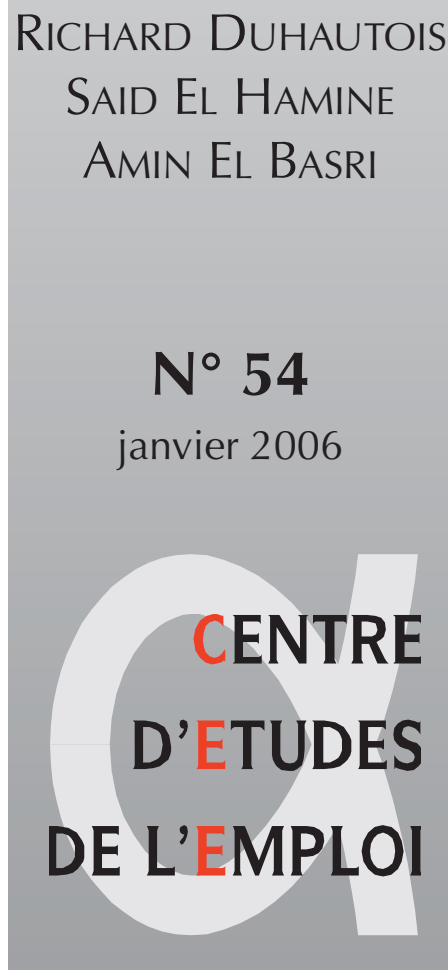

«LE DESCARTES I»

29, PROMENADE MICHEL SIMON

93166 NOISY-LE-GRAND CEDEX

TÉL. 0I $45926800 \quad$ FAX OI 49310244

MÉL. cee@cee.enpc.fr

http://www.cee-recherche.fr 


\title{
Croissance de la productivité et réallocations dlemplois au Maroc : la contribution des créations et disparitions dlentreprises
}

\author{
RICHARD DUHAUTOIS \\ richard.duhautois@cee.enpc.fr \\ Centre d études de l emploi, Crest \\ SAID EL HAMINE \\ AMIN EL BASRI \\ Ministère du Commerce et de l'Industrie (Maroc)
}

\section{DOCUMENT DE TRAVAIL}

$$
N^{\circ} 54
$$


ISSN 1629-7997

ISBN 2-11-095787-5 


\title{
CROISSANCE DE LA PRODUCTIVITÉ ET RÉALLOCATIONS D EMPLOIS AU MAROC : LA CONTRIBUTION DES CRÉATIONS ET DISPARITIONS DENTREPRISES
}

\author{
Richard Duhautois, Said El Hamine, Amin El Basri
}

\begin{abstract}
RESUME
Dans cet article, nous nous proposons didentifier les différents types $\mathrm{d}$ entreprises (entreprises pérennes, entreprises apparues ou disparues) qui contribuent à la croissance de la productivité du travail et de la productivité globale des facteurs (PGF) dans 1 industrie marocaine. Nous utilisons deux méthodes de décomposition (Griliches, Regev [1995]; Foster, Haltiwanger et Krizan [1998]) qui permettent de prendre en compte le processus de destruction créatrice $\square$ le renouvellement des entreprises $\square$ et la croissance des entreprises pérennes. Ensuite, nous mettons la croissance de la productivité en relation avec les réallocations demplois, c est-à-dire avec les créations et les destructions brutes d emplois.

La période étudiée est divisée en deux : 1990-1995 et 1995-2000. En outre, nous distinguons les dynamiques sectorielles (au sein de 1industrie), car les effets de recomposition de 1êconomie marocaine ont des conséquences à la fois sur la productivité et 1 emploi.

Nous montrons, dans un premier temps, que la croissance de la productivité du travail est fortement dépendante de la croissance au sein des entreprises pérennes; dans un second temps, que, pour la croissance de la productivité globale des facteurs, les entrées jouent un rôle plus important, notamment en incorporant du capital plus performant; enfin, que les disparitions d entreprises expliquent la différence de croissance de 1๔emploi entre les deux périodes de la décennie 90.
\end{abstract}

Mots-clefs : productivité, décomposition, démographie des entreprises, Maroc, réallocations d厄emploi.

JEL : D21 $\square$ D24 $\square 23 \square$ O55 


\title{
Productivity Growth and Gross Job Flows in Morocco
}

\begin{abstract}
This article tries to identify the different types of firms (survival, entry and exit firms) which contribute to labour and total factor productivity growth in the Moroccan manufacturing industries. We use two decomposition methods (Griliches, Regev [1995]; Foster, Haltiwanger and Krizan [1998]) which enable us to take into account the creativedestruction process and the evolution of survivals. Afterwards, we put in relation these decompositions with gross job flows (gross creation and destruction).

The data cover the 90 $\mathrm{s}$ and the period is divided into two: 1990-1995 and 1995-2000. In the manufacturing industry, we are able to distinguish sectoral dynamics and we show that modifications of Moroccan economy have impact on productivity and employment.

We show: First, labour productivity in Morocco changes with the dynamics of productivity within survival firms; Second, total factor productivity depends on entrants; Third, exits of firms during the last period explain the lower job growth rate.
\end{abstract}

Key words: productivity, decomposition, fims, entry and exit of firms, Morocco, gross job flows. 


\section{INTRODUCTION}

L observation économique au sein des secteurs dactivités $\square$ quelque soit la nomenclature utilisée $\square$ montre qu ill existe une forte hétérogénéité entre entreprises (Caves, 1998). Ainsi, même dans les secteurs à forte croissance, des entreprises disparaissent et dans les secteurs en déclin, certaines sont florissantes. De la même façon, les périodes de croissance et de récession macro-économiques n engendrent pas forcément des évolutions pro-cycliques pour toutes les entreprises. L hétérogénéité des comportements des entreprises au sein de chaque secteur et dans le cycle économique a des implications importantes sur leẻvolution agrégée des principaux agrégats macro-économiques. Les études à partir de données individuelles ${ }^{1}$ d entreprises montrent que les comportements hétérogènes ne sont pas sans influence sur le niveau agrégé et, de ce fait, limitent les extrapolations à partir dune entreprise considérée comme représentative. Les disparitions et les créations d entreprises sont relativement nombreuses chaque année et sont directement des éléments clés de 1 évolution macroéconomique (Crépon et Duhautois, 2004).

Dans cet article nous nous concentrons sur la dynamique de la croissance de la productivité au Maroc, expliquée par les données individuelles. On se propose de caractériser deux périodes de la décennie 1990 par la différence de leurs effets sur 1๔emploi et la productivité. On utilise pour cela une méthode de décomposition originale de la productivité apparente du travail et de la productivité globale des facteurs (Baily, Hulten et Campbell, 1992 ; Foster, Haltiwanger et Krizan, 1998; Griliches et Regev, 1995). La croissance de la productivité pour différents types dentreprises (entreprises présentes tout au long de la période dites entreprises pérennes, entreprises apparues ou au contraire disparues (Baldwin, 1995 ; Ahn, 2001)) est mise en relation avec les réallocations d emplois, c est-à-dire avec les créations et les destructions brutes ${ }^{2}$ demplois. La période étudiée est divisée en deux : 1990-1995 et 1995-2000. On distingue les dynamiques sectorielles (au sein de lindustrie), car les effets de recomposition de 1économie marocaine ont des conséquences à la fois sur la productivité et 1 emploi.

Au cours de ces deux périodes, 1 êvolution de la productivité est-elle comparable ou au contraire nettement différenciée? Quel est le rôle respectif des créations et disparitions d entreprises et des entreprises pérennes dans 1 évolution de cette productivité ? Quel est le lien entre 1Ґévolution de la productivité, et celle de 1Ґemploi ? Celle-ci est elle imputable aux créations et disparitions, ou bien aux entreprises pérennes? Cette répartition est-elle susceptible daassurer au système productif une croissance « schumpetérienne », c est-à-dire le renouvellement des entreprises par destruction créatrice et les technologies innovantes associées (Jovanovic, 1982 ; Ericson et Pakes, 1995). Les données individuelles dentreprise utilisées pour avancer quelques éléments de réponse à ces questions sont issues de la base de données de 1厄enquête annuelle sur les industries de transformation.

\footnotetext{
${ }^{1}$ Pour 1厄emploi : Davis et Haltiwanger, 1990, 1992, 1998 ; Duhautois, 2002 ; pour 1linvestissement : Doms et Dunne, 1998 ; Caballero, Engel et Haltiwanger, 1995; Duhautois et Jamet, 2002; pour la productivité : Baily, Hulten et Campbell, 1992 ; Bartelsman et Doms, 2000 ; Crépon et Duhautois, 2004.

${ }^{2}$ La croissance de la productivité agrégée est décomposée au moyen des méthodes de Griliches et Regev (1995) et de Foster, Haltiwanger et Krizan (2000). Pour les flux bruts demplois, on utilise la méthode de Davis et Haltiwanger (1992, 1999).
} 


\section{Encadré 1 \\ Quelques faits sur léconomie marocaine et les industries de transformation}

La croissance économique s'est « essoufflée » à partir de 1990 (+ 2,4 \% en moyenne sur la décennie) en raison de facteurs conjoncturels telles les périodes de sécheresse et surtout la persistance de problèmes structurels. Beaucoup d'entrepreneurs locaux ont vendu leurs entreprises à de grands groupes internationaux plutôt que de chercher à les moderniser : par exemple AXA a racheté la Compagnie africaine d'assurances, Danone le biscuitier Bisco, Coca-Cola les embouteilleurs de Fès et de Marrakech. Cette situation explique pourquoi les investissements directs étrangers (IDE) ont souvent été le résultat d'opérations de portefeuille et de privatisations et non d'investissements nouveaux. Près de la moitié des 15,9 milliards de dirhams ${ }^{3}$ (DH) de recettes générées par les privatisations sont le fruit d'acquisitions par des entreprises étrangères. La dette extérieure du Maroc $\mathrm{s}$ est récemment réduite. Toutefois cette diminution a été en partie compensée par un alourdissement de la dette interne, qui a grimpé de $38 \%$ à $47 \%$ du PIB entre 1996 et 2000.

La décennie 90 a été marquée par deux cycles, comme dans les pays dEurope occidentale. La première période qui seêtale de 1991 à 1994 a vu une décélération de la croissance. Alors que la deuxième, allant de 1994 à 1998, a connu un début de relance de 1iindustrie, concrétisée par la progression des investissements de $7 \%$ contre $1 \%$ pour la période 1991-1994, la croissance de la valeur ajoutée au taux de $7 \%$ au lieu de $6 \%$ et 1 êvolution de 1 effectif au rythme annuel moyen de $2,5 \%$ contre $1 \%$.

Pour ce qui est du commerce extérieur, les importations ont enregistré au cours de la période 19911994 une croissance annuelle moyenne de près de $3 \%$, au moment où les exportations ont accusé une légère chute en moyenne de $0,5 \%$, ce qui a contribué à une dégradation de la position de la balance commerciale avec un taux de couverture moyen de près de $57 \%$ et un déficit commercial denviron 27 milliards de DH. Concernant la période 1994-1998, la situation des transactions commerciales hors admissions temporaires a été caractérisée par une, progression annuelle moyenne de $6 \%$ pour les importations et $5 \%$ pour les exportations. La position de la balance commerciale s est soldée par un déficit moyen de près de 30 milliards de $\mathrm{DH}$, et un taux de couverture moyen d environ $57 \%$ sur cette période.

Par ailleurs, et en dépit dune légère diversification, les exportations industrielles sont concentrées sur 20 destinations dont 8 font partie de 1Union européenne et portent à plus de $75 \%$ sur 20 principaux produits.

La période 1998-2000 a été caractérisée par une croissance annuelle moyenne ne dépassant pas les $1 \%$ pour la majorité des indicateurs macro-économiques, à 1๔xception de la valeur ajoutée qui a enregistré un taux de croissance annuel moyen de $2 \%$ et de 1 investissement qui s est accru au taux de $10 \%$. La croissance de la valeur ajoutée en volume des industries de transformations au Maroc a atteint environ $23 \%$ entre 1990 et 1995 et $16 \%$ entre 1995 et 2000 . La valeur ajoutée de ces industries représente $16 \%$ du PIB marocain en 2000 (14\% en 1990). L emploi augmente de 410000 salariés en 1990 à $495=000$ salariés en $2000(75 \%$ de la croissance de 1 emploi concerne 1 emploi féminin).

Les industries textiles et du cuir - industrie la plus importante - emploient environ $40 \%$ de la main$\mathrm{d} \square$ uvre des industries de transformations en 1990 et en 2000. Pendant la récession du début des années 90, 1匹mploi de ce secteur a stagné et a commencé à redémarrer à partir de 1995, date de la reprise. Depuis 1998, le secteur connaît à nouveau des difficultés propres au secteur. Le second secteur important en termes de main-d $\square$ uvre est le secteur chimique et parachimique qui emploi environ un quart de la main-d $\square$ uvre. En 2000, environ $20 \%$ des salariés des industries de transformations sont des saisonniers et cette part a diminué tout au long de la décennie 90 .

\footnotetext{
310 dirhams sont environ équivalents à 1 euro
} 


\section{PRODUCTIVITÉ ET DÉMOGRAPHIE DES ENTREPRISES : QUELQUES ÉLÉMENTS EMPIRIQUES}

La croissance économique implique invariablement des réallocations de facteurs de production. Les réallocations de facteurs peuvent être externes et internes au marché. Lorsqu elles sont externes, on parle de destruction créatrice. Les modèles de destruction créatrice (Caballero et Hammour, 1994 ; Campbell, 1997) mettent 1accent sur le rôle des entrées et des sorties des entreprises. Si les nouvelles technologies ne peuvent être adoptées que par les nouveaux entrants, la croissance se produit par les entrées et sorties, ce qui nécessite des réallocations dinputs. Lorsque les réallocations sont internes, les modèles (Cooper, Haltiwanger et Power, 1997) mettent 1 accent sur le fait que les établissements présents sur un marché peuvent remplacer les anciennes technologies par de nouvelles. C est ce changement de technologie qui peut engendrer des réallocations demplois au sein du même établissement et entre établissements. Par exemple, une amélioration technologique dans un établissement peut apporter des changements, à la fois, dans le niveau de 1๔mploi et dans le niveau des qualifications.

Bien que les économistes se soient toujours intéressés aux créations et aux disparitions $\mathrm{d}$ entreprises, les données permettant de bien les quantifier ne sont accessibles que depuis peu de temps. Des résultats importants concernant le lien entre démographie des entreprises et productivité sont obtenus à partir de LRD (longitudinal research database) (Mac Guckin et Stiroh, 1999). Ils montrent notamment que la croissance de la productivité aux États-Unis entre 1963 et 1992 dépend de linteraction entre les entreprises en place qui survivent et les nouveaux entrants. Aux Pays-Bas, cette interaction étudiée dans le secteur des services aux entreprises est confirmée sur la période 1987-1995 (Van der Wiel, 1999). Un des résultats fondamentaux est 1 limportance des vieilles et nouvelles entreprises dans la croissance de la productivité du travail. Deux effets différents engendrent la croissance de la productivité : un premier effet que nous traduisons «effet millésime» («Vintage effect»), qui mesure la productivité des nouveaux entrants relativement aux nouveaux entrants des périodes précédentes : les nouvelles entreprises entrent sur un marché avec un niveau d expertise plus important, des technologies plus performantes ou une organisation plus adéquate; un deuxième effet $1 \varangle$ effet vieillissement » («Survival effect »), qui mesure la productivité des entreprises en place qui ont bénéficié des effets dapprentissage et d économies d échelle. Aux États-Unis, les gains de 1๔effet millésime sont estimés à 2\% par an entre 1963 et 1992 dans 1 industrie (Mac Guckin et Stiroh, 1999). Leffet vieillissement augmente avec lâge de 1entreprise: par exemple, la productivité des entreprises survivantes en 1992, dont la création a été effectuée entre 1963 et 1967, a augmenté de 2,3\% par an.

Leffet millésime et 1๔effet vieillissement sont clairement des sources importantes de la croissance de la productivité. Toutefois, le rôle de ces deux effets n est pas identique dans les différentes phases du cycle économique. On sait que la productivité du travail diminue pendant les récessions et augmente pendant les phases de croissance (Baily, Bartelsman et Haltiwanger, 1996). La procyclicité de la productivité peut $\mathrm{s}$ expliquer par plusieurs facteurs dont les rendements croissants, le labour hoarding, les coûts d ajustement et les externalités positives: les rendements $\mathrm{d}$ êchelle croissants impliquent qu une augmentation du volume des facteurs engendre une augmentation plus grande de la production. En phase de croissance, 1 utilisation de main- $\mathrm{d} \square$ uvre supplémentaire augmente la production plus que proportionnellement. Le déclin de la production pendant les récessions mène naturellement à la baisse de la productivité globale des facteurs et, en fonction des paramètres de la fonction de production de 1entreprise, à la baisse de la productivité du travail ; le labour hoarding 
implique que les firmes gardent leurs salariés quelle que soit la conjoncture afin de préserver le capital humain. Pour que les entreprises pratiquent le labour hoarding, il faut qu elles anticipent un retournement de la conjoncture - une hausse de la demande de travail - dans le futur. Le labour hoarding est dautant plus faible que le niveau d emploi anticipé est faible ; dans la continuité, il existe des coûts $\mathrm{d}$ ajustement à réorganiser le processus de production lors des chocs conjoncturels. Cest seulement à long terme, si le choc persiste, que les entreprises $\mathrm{s}$ adaptent; la productivité est plus grande lorsque toutes les entreprises connaissent une croissance de la production. Une des implications de cela est que 1 iimpact $\mathrm{d}$ un choc sur la productivité $\mathrm{d}$ une entreprise dépend de comment il frappe les autres entreprises.

En outre, les recherches récentes à partir de données individuelles ont conduit à de nombreux concepts et à de nombreuses mesures permettant une meilleure compréhension des évolutions agrégées. Notamment, les réallocations de facteurs de production peuvent jouer un rôle important dans la croissance de la productivité agrégée (Foster, Haltiwanger et Krizan, 1999).

\section{LES DONNÉES}

Les données utilisées dans ce travail sont issues de la base de 1๔enquête annuelle sur les industries de transformation marocaines. Cette enquête est menée de façon périodique et permanente par le Département du Commerce et $d$ Industrie auprès de la totalité des entreprises du secteur manufacturier marocain depuis 1985. Elle se veut exhaustive mais il existe un secteur informel assez développé qui ne permet pas de recenser 1匹nsemble des entreprises. Le fichier fournit des informations sur toutes les entreprises industrielles quels que soient le chiffre daffaires et la taille de 1 entreprise. Ce fichier, qui est 1 unique source $\mathrm{d}$ information sur le secteur industriel au Maroc, couvre 1ensemble des branches industrielles et 1 ensemble des régions territoriales et contient un très grand nombre de petites et moyennes entreprises : la taille médiane est de 20 salariés et $92 \%$ des entreprises ont moins de 200 salariés (cf. annexe, tableau 5, pour une distribution plus complète). Ces chiffres sont différents de ce qu on trouve en France par exemple car il existe un secteur informel très développé au Maroc. Ainsi, certaines petites entreprises échappent au recensement du ministère de 1 industrie.

La période étudiée (1990-2000) est une période cruciale dans le développement économique du Maroc. En effet, elle vient juste après 1 application du programme dajustement structurel (PAS) de 1983-1989 et la période du début effectif de 1øuverture du Maroc avec la signature de 1 accord $\mathrm{d}$ association avec 1 Union européenne et 1 instauration $\mathrm{d}$ une zone de libre échange en 1995.

Dans ce travail, on retient 1 entreprise et non 1êtablissement comme unité de base pour le calcul des flux. A priori, les analyses effectuées au niveau de 1๔entreprise ou au niveau de 1 êtablissement sont assez différentes (Davis et Halltiwanger, 1998). En effet, il peut exister des flux entre établissements au sein dune même entreprise. Cependant, les taux de création $\mathrm{d}$ emplois sont pratiquement identiques en moyenne pour les petites entreprises, que $1 \curvearrowleft$ n utilise comme unité les entreprises ou les établissements (Klette et Matissen, 1996).

Les données aberrantes de certaines entreprises ont conduit à les exclure : tel est le cas des entreprises ayant été estimées au moins deux fois des trois dates 1990, 1995 et 2000. Au contraire, on a implémenté des informations pour environ $10 \%$ des entreprises en 1995 car elles existaient en 1990 et en 2000. La technique dimplémentation est la suivante : on a 
récupéré 1 année la plus proche de 1995 dans les fichiers dorigine pour la simuler. Lexclusion de certaines entreprises sur un échantillon relativement petit peut conduire à modifier les résultats mais il est impossible de les garder étant donné le poids de certaines entreprises aberrantes.

Au final, on dispose de 3392 entreprises en activité en 1990 et 1995, dites entreprises «pérennes »; de 1910 entreprises en activité en 1990 et qui ont disparu en 1995, qu on appelle les entreprises «sortantes »; de 1468 entreprises en activité en 1995 et qui étaient inexistantes en 1990, qu on appelle les entreprises «entrantes». Entre 1995 et 2000, les chiffres sont respectivement 3351 entreprises pérennes, 1468 entreprises sortantes et 1973 entrantes.

On calcule la productivité apparente du travail comme le rapport entre la valeur ajoutée en volume et 1effectif total (permanent plus saisonnier). Le rapport moyen sur la décennie 1990 entre permanent et saisonnier est d environ 1,2 : elle atteint 1,26 entre 1990 et 1997 et 1,1 entre 1998 et 2000. La productivité calculée n est pas une productivité car nous ne disposons pas du nombre d heures pour les permanents. On dispose de la totalité des heures travaillées pour les travailleurs saisonniers et nous ramenons à une quantité de travailleurs en divisant par le nombre de jours ouvrables. La valeur ajoutée est déflatée par lindice des prix à la consommation qui est le seul indice dont nous disposons. La productivité globale des facteurs (PGF) est égale au rapport de la valeur ajoutée en volume sur les facteurs de production, capital et travail. La part du facteur travail est de $0,7^{4}$ et celle du capital de 0,3 . Le travail est toujours 1 effectif total. Dans le fichier, nous ne disposons pas du stock de capital (les immobilisations des entreprises). En revanche, on a 1 investissement annuel des entreprises. Ainsi, on utilise les investissements pour reconstruire le stock de capital. Comme base de départ, on utilise le capital social de 1ॅentreprise qui, lui est disponible; il sert de distribution au de base pour reconstruire le stock de capital chaque année. Les prix de linvestissement ne sont pas disponibles tels quels au Maroc. Par conséquent le capital est déflatée par un indice qui représente la même différence que liindice des prix de la valeur ajoutée et ceux de 1 investissement en France.

\section{LES MÉTHODES}

\subsection{Les décompositions de productivité}

La croissance agrégée de la productivité peut être décomposée en trois éléments : la croissance de la productivité au sein des entreprises et deux éléments qui ont trait aux réallocations de facteurs de production et de la valeur ajoutée. Ces réallocations correspondent à la fois aux entreprises présentes tout au long de la période, et au processus de destruction-créatrice des entreprises. Plusieurs méthodes permettent daboutir à une telle décomposition.

\footnotetext{
${ }^{4}$ Nous avons estimé à 0,68 la part du travail par MCO que $1 \sqsubset$ on a arrondi à 0,7 .
} 
La productivité pour l'ensemble de l'économie à la date $\mathrm{t}, P_{t}$ peut s'écrire comme une moyenne pondérée de la productivité de chaque entreprise :

$$
P_{t}=\sum_{i} \theta_{i t} p_{i t}
$$

où $\theta_{i t}$ est la part de l'emploi de l'entreprise i à la date $\mathrm{t}$ dans l'emploi total et $p_{i t}$ une mesure de la productivité de cette entreprise.

La première décomposition, proposée par Baily, Hulten et Campbell (1992), notée par la suite BHC, découle directement de l'identité :

$$
\Delta P_{t}=\sum_{i \in S} \theta_{i t-k} \Delta p_{i t}+\sum_{i \in S} \Delta \theta_{i t} p_{i t}+\sum_{i \in N} \theta_{i t} p_{i t}-\sum_{i \in X} \theta_{i t-k} p_{i t-k} \text { (BHC) }
$$

où $S, N$ et $X$ représentent respectivement les entreprises présentes en début et en fin de période (entreprises dites survivantes : elles existent en $t-k$ et en $t$ ), et celles qui entrent et qui sortent du marché entre t-k et t. Le premier terme du membre de droite de l'équation est la variation de productivité qui découle de l'évolution de la productivité à liintérieur des entreprises survivantes ; c'est l'effet « intra » ${ }^{5}$. Le deuxième terme constitue l'effet « inter » des entreprises survivantes : autrement dit, cet effet exprime la variation de productivité induite par un changement de composition. Le troisième et le quatrième terme sont les effets des entrées et des sorties sur la croissance de la productivité ; la différence entre ces deux termes est l'effet net de l'entrée.

La décomposition $\mathrm{BHC}$ peut poser deux problèmes quant à la contribution des entrées et des sorties. Haltiwanger (1997) montre que si, à productivité égale, la part des entrants est plus faible que celle des sortants, l'effet net de l'entrée peut être négatif simplement par le fait quill y a plus de sortants (cf. supra). En outre, si on s'intéresse au signe des contributions (brutes), cette décomposition des entrées et sorties leur donne un signe positif, alors que les entreprises qui entrent et qui sortent d'un secteur ont une productivité du travail inférieure aux moyennes sectorielles.

Foster, Haltiwanger et Krizan (1998) proposent une méthode de décomposition (notée FHK) de la productivité qui lève ces incertitudes sur la contribution des entrées et sorties. La décomposition est la suivante :

$$
\begin{aligned}
& \Delta P_{t}=\sum_{i \in S} \theta_{i t-k} \Delta p_{i t}+\sum_{i \in S} \Delta \theta_{i t}\left(p_{i t-k}-P_{t-k}\right)+\sum_{i \in S} \Delta \theta_{i t} \Delta p_{i t} \\
& +\sum_{i \in N} \theta_{i t}\left(p_{i t}-P_{t-k}\right)-\sum_{i \in X} \theta_{i t-k}\left(p_{i t-k}-P_{t-k}\right)
\end{aligned}
$$

Le premier terme est 1 effet intra déjà rencontré dans $\mathrm{BHC}$, le deuxième terme est un effet inter et le troisième terme est un effet de covariance. Une augmentation de 1 emploi conduit à un effet inter positif si 1匹entreprise à une productivité plus élevée que la productivité moyenne initiale. L'effet de covariance est positif lorsque la productivité et l'emploi évoluent dans le même sens. Le quatrième et le cinquième terme sont les effets de l'entrée et de la sortie du marché.

La décomposition FHK a l'avantage de mesurer les contributions des entrées/sorties relativement à la productivité globale moyenne : ces effets sont négatifs pour la productivité du travail, dans la mesure où les entreprises récentes et celles qui sont sur le point de sortir ont des productivités inférieures à la moyenne. L'effet de la covariance entre la productivité et l'emploi limite mécaniquement la dimension inter de l'évolution de la productivité et est souvent négatif car les entreprises dont la productivité du travail augmente sont fréquemment

\footnotetext{
${ }^{5}$ Cet effet est l'effet interne de Disney et al.
} 
celles où les effectifs ont baissé ex ante. En revanche, les auteurs le soulignent eux-mêmes, cette décomposition est sensible aux erreurs de mesure de l'emploi.

Un moyen de lisser les erreurs de mesure est d'utiliser la méthode de Griliches et Regev (1995), notée GR :

$$
\begin{array}{r}
\Delta P_{t}=\sum_{i \in S} \bar{\theta} \Delta p_{i t}+\sum_{i \in S} \Delta \theta_{i t}\left(\overline{p_{i}}-\bar{P}\right) \\
+\sum_{i \in N} \theta_{i t}\left(p_{i t}-\bar{P}\right)-\sum_{i \in X} \theta_{i t-k}\left(p_{i t-k}-\bar{P}\right)
\end{array}
$$

Le premier terme représente toujours la contribution intra des survivants, pondérée par la moyenne temporelle de la part de l'emploi de l'entreprise i. Les autres termes $\square$ la contribution inter et les contributions des entrées-sorties $\square$ se calculent relativement à la moyenne. Cette méthode a le mérite de rendre les décompositions de productivité moins sensibles aux erreurs de mesure mais rend l'interprétation des contributions plus délicates. Notamment, l'effet intra dépend d'une certaine façon de l'effet inter puisqu'il prend en compte la moyenne temporelle des parts.

Ces trois décompositions ont chacune leurs mérites et leurs inconvénients, au regard de 1ळbjectif poursuivi : décomposer la croissance de la productivité entre trois effets « intra», « inter» et « entrées nettes », ce dernier ayant des composantes entrées et sorties négatives. Le défaut de la décomposition BHC réside dans 1 expression des entrées-sorties, dont chacune de deux composantes est alors positive. Elle ne donne que les productivités moyennes pondérées des entrants et des sortants. Le problème de la décomposition (FHK) concerne l'interprétation du terme croisé (la covariance de la productivité et des parts d'emplois). Ce terme est vraisemblablement toujours négatif. Enfin, la décomposition (GR) ne différencie pas rigoureusement effets intra et inter. On a retenu ici les méthodes (FHK) et (GR), à 1 exclusion de la méthode (BHC).

\subsection{Les flux bruts demplois}

Les définitions des flux d emplois et la méthode de calcul de ces flux, ainsi que les taux associés, utilisées dans la plupart des études s inspirent de Davis et Haltiwanger (1990). On peut également décomposer les flux en une moyenne pondérée permettant $\mathrm{d}$ en différencier les éléments constitutifs. Les deux méthodes utilisées ici sont détaillées dans ce qui suit. Le taux de création brute $\mathrm{d}$ emplois (TC) à la date $t$ est égal à la somme des emplois créés entre $t-1$ et $t$.

$$
T C_{t}=\sum_{i \in S^{+}} \frac{\left(e_{i t}-e_{i t-1}\right)}{Z_{t}}=\sum_{i \in S^{+}} \omega_{i t} \dot{e}_{i t}
$$

où $S^{+}$est 1 ensemble des entreprises i dont la variation de 1 emploi e a été positive entre $t-1$ et $t$, i.e $\dot{e}_{i t}>0$, avec $Z_{t}=0,5\left(e_{t}+e_{t-1}\right), \omega_{i t}=\left(e_{i t}+e_{i t-1}\right) /\left(e_{t}+e_{t-1}\right)$ et $\dot{e}_{i t}=\left(e_{i t}-e_{i t-1}\right) / 0,5\left(e_{i t}+e_{i t-1}\right)$. 
La destruction brute $\mathrm{d}$ emplois à la date $t$ est égale à la somme des emplois détruits entre $t-1$ et $t$.

$$
T D_{t}=\sum_{i \in S^{-}} \frac{\left(e_{i t}-e_{i t-1}\right)}{Z_{t}}=\sum_{i \in S^{-}} \omega_{i t} \dot{e}_{i t}
$$

où $S^{-}$est le sous ensemble des entreprises i dont la variation de 1๔emploi a été négative entre $t-1$ et $t$ i.e $\dot{e}_{i t}<0$.

Le taux de croissance nette de 1๔emploi est :

$$
\dot{e}_{t}=\sum_{i} \omega_{i t} \dot{e}_{i t}=\sum_{i \in S^{+}} \omega_{i t} \dot{e}_{i t}+\sum_{i \in S^{-}} \omega_{i t} \dot{e}_{i t}=T C_{t}+T D_{t}
$$

Le taux de réallocation brute demploi à la date $t$ est défini par la différence des taux de créations brutes et des destructions brutes demplois entre $t-1$ et $t$.

$$
T R_{t}=\sum_{i \in S^{+}} \omega_{i t} \dot{e}_{i t}-\sum_{i \in S^{-}} \omega_{i t} \dot{e}_{i t}=T C_{t}-T D_{t}
$$

Afin danalyser plus finement les variations d emplois entre entreprises pérennes et non pérennes, on décompose le taux de croissance globale $\dot{e}$. En nommant les nouvelles entreprises (c), les entreprises pérennes créatrices d emplois (cp), les entreprises pérennes destructrices demplois (dp) et les disparues (d), on obtient :

$$
\begin{aligned}
& \dot{e}=\sum_{i} \omega_{i} \dot{e}_{i}=\sum_{c} \omega_{i} \dot{e}_{i}+\sum_{c p} \omega_{i} \dot{e}_{i}+\sum_{d p} \omega_{i} \dot{e}_{i}+\sum_{d} \omega_{i} \dot{e}_{i}, \\
& \dot{e}=2\left(\sum_{c} \omega_{i}\right)+\left(\sum_{c p} \omega_{i}\right) \dot{e}_{c p}+\left(\sum_{d p} \omega_{i}\right) \dot{e}_{d p}-2\left(\sum_{d} \omega_{i}\right)
\end{aligned}
$$

\section{RÉSULTATS}

La croissance de la productivité agrégée est composée de trois éléments : la croissance de la productivité au sein des entreprises pérennes (composante « intra ») et deux éléments qui ont trait à la réallocation des facteurs de production entre entreprises pérennes (composante « inter») et entre entreprises qui se créent ou qui disparaissent (effet net de 1๔entrée). La composante intra est souvent associée soit au progrès technique, soit au « learning by doing » alors que le processus de réallocation (de 1ऍmploi et de la valeur ajoutée) refléterait plutôt 1Ґêvolution du marché (réallocations de facteurs entre entreprises).

Leffet intra dépend des changements dans la quantité et la qualité des facteurs de production et de 1 intensité avec laquelle ils sont utilisés dans le processus productif. À court terme, c est lintensité qui varie pour faire évoluer la productivité.

Leffet inter reflète les gains de productivité des entreprises en place qui gagnent des parts de marchés dans leur secteur mais aussi 1 évolution de la demande qui fluctue dun secteur à 1 autre. L effet net des créations et disparitions d entreprises représente 1 impact de la contribution des nouvelles entreprises à la croissance de la productivité relativement aux sorties dentreprises.

Comme on 1 a souligné, il existe plusieurs décompositions de la variation de la productivité entre ses trois composantes (intra, inter et effet net de 1匹entrée). Lapplication de ces méthodes de décomposition aux données $\mathrm{d}$ entreprise permet de quantifier les contributions à 1 êvolution de la productivité, de chacune des catégories d entreprises (entreprises pérennes, 
entreprises apparues et entreprises disparues au cours de la période). Ce calcul est présenté pour la productivité apparente du travail et pour la productivité globale des facteurs $\left(\mathrm{PGF}^{6}\right)$, et porte sur chacune des deux périodes.

La productivité apparente du travail a augmenté de 5,6\% entre 1990 et 1995 et de 2,8\% entre 1995 et 2000 (cf. annexe, tableau 1-A). La composante intra, quelle que soit la méthode et quelle que soit la période, représente pratiquement à chaque fois plus de la totalité de la croissance de la productivité. La composante inter, qui est la composante liée aux réallocations demplois et de valeur ajoutée, contribue pour environ la moitié de la composante intra. Certaines entreprises ont donc connu une évolution positive de 1 emploi en ayant des productivités inférieures à la productivité moyenne. Le terme de covariance est négatif, ce qui est conforme à liintuition. L effet des entrées nettes est légèrement positif sur les deux périodes. Au total, la différence de croissance de productivité apparente du travail que 1 on observe dans le temps est liée fortement à la croissance au sein des entreprises pérennes.

La PGF a diminué de -1,6 \% entre 1990 et 1995 et augmenté de 1,5\% entre 1995 et 2000 (cf. annexe, tableau 1-B). La composante intra est en phase avec la croissance totale de la PGF. Ce phénomène est dû au non-ajustement du stock de capital dans les entreprises pérennes : si à court terme (même avec un léger délai) on peut ajuster le facteur travail à 1évolution de la demande, le capital est quasi-fixe. Sa contribution à la variation de la productivité du travail lui est inférieure en valeur absolue (résultat que 1ळon retrouve dans toutes les études).

La hiérarchie des nouvelles entreprises et des entreprises en cessation, suivant leur contribution à 1 évolution globale de la productivité, $\mathrm{n}$ est pas tout à fait la même selon que 1 on considère la productivité du travail ou la productivité globale. S agissant de la productivité du travail, les entrées brutes (avant dernière colonne) ont une contribution constamment négative au cours de chacune des quatre périodes, au contraire des sorties du marché dont 1 limpact $\mathrm{s}$ avère positif: les entreprises en création et en cessation ont une productivité du travail plus faible que les pérennes, liimpact net positif résultant de la plus grande faiblesse de la productivité des entreprises qui sortent du marché. Leffet des entrées semble davantage lié que celui des cessations à la variation cyclique, au moins pour la méthode FHK. Dans le cas de la PGF, les contributions des entrantes sont plus faibles. Les entreprises créées et disparues ont une productivité légèrement inférieure à la moyenne. En effet, les entreprises, à mesure que leur ancienneté augmente, utilisent de plus en plus de capital relativement au travail (la croissance de la productivité du travail est corrélée positivement au stock de capital), c est pourquoi la productivité apparente du travail croît beaucoup et la PGF moins dans les entreprises pérennes les plus jeunes.

Cette décomposition peut être réalisée par sous-secteur (cf. annexe, tableau 2). Quel que soit le sous-secteur, 1๔effet intra représente toujours la majeure partie de la croissance de la productivité du travail et une partie importante de la PGF. Cette proportion est plus forte que celle obtenue par Disney et al. (2000) sur données anglaises (avec un effet intra d environ $50 \%$ pour la productivité du travail) entre 1980 et 1992. En revanche, ils sont proches de ceux trouvés par Foster, Haltiwanger et Krizan (2000) sur données américaines entre 1977 et 1987, lorsquills utilisent 1 emploi comme pondération.

Dans tous les trois premiers sous-secteurs, la croissance de la productivité du travail est plus faible entre 1990 et 1995 qu entre 1995 et 2000. Seul le secteur Textile et Cuir connaît une

\footnotetext{
${ }^{6}$ La pondération utilisée est 1๔emploi également. On peut également utiliser la valeur ajoutée ou un facteur composite du travail et du capital.
} 
évolution inverse. Dans pratiquement tous les secteurs, 1 impact du processus de destruction créatrice est positif.

Ce qu on cherche à voir dans la suite concerne 1 limpact sur les réallocations d emplois des mouvements liés au processus de créations et disparitions dentreprises.

Le taux de croissance globale nette de 1匹emploi peut être décomposé en quatre facteurs additifs : la part de cette croissance imputable aux créations, celle qui résulte des disparitions, et celle qui incombe aux entreprises pérennes, en distinguant, parmi ces dernières, celles qui créent des emplois de celles qui en détruisent.

Le taux de croissance nette de 1๔emploi sur la période 1995-2000 a été de 2,1\%, plus faible que sur la période 1990-1995 où il était de 6,9\% (cf. annexe, tableau 3). Ce taux de croissance nette de 1 emploi est associé à des taux de créations et de destructions brutes $\mathrm{d}$ emplois respectivement de $34,1 \%$ et $27,2 \%$, chiffres comparables à ce qu on peut trouver dans la littérature sur les créations et destructions d emplois ${ }^{7}$; au cours de la seconde période, ils sont égaux à $35 \%$ et $32,9 \%$. On vérifie tout d abord le fait couramment admis que les créations d entreprises apportent une contribution importante aux créations brutes d emplois (Davis et Haltiwanger, 1998 [pour les États-Unis]; Duhautois, 2002 [pour la France]). Ainsi, pour la période 1990-1995, le taux de créations global de 34,1\% se décompose en 17,3\% imputable aux entreprises qui se créent (c est-à-dire qui apparaissent en 1991, 1992, 1993, 1994 et 1995) et 16,8\% imputable aux entreprises pérennes (cf. annexe, tableau 4). Lemploi s accroît à un rythme plus rapide dans les entreprises pérennes entre 1995 et 2000 quentre 1990 et 1995.

Les destructions d emplois ont augmenté de près de 5 points sur la seconde période. Deux effets sont à $1 \square$ uvre: dune part, une stagnation des destructions dans les entreprises pérennes (de $\square 0,5 \%$ à $-10,6 \%$ ) et dautre part, une augmentation des destructions dans les entreprises qui disparaissent (de $\square 6,7 \%$ à $\square 22,2 \%$ ).

$\mathrm{Au}$ total, le contraste entre les deux périodes est double : dune part, dans les entreprises pérennes, les créations d emplois sont plus importantes pendant la seconde période alors que les destructions sont stables. D autre part, la contribution des créations $\mathrm{d}$ entreprises à 1 augmentation de 1๔emploi accuse un léger retrait pendant la seconde période, tandis que la contribution des destructions est plus importante. Ainsi, ce sont surtout les disparitions $\mathrm{d}$ entreprises qui expliquent la différence de croissance de 1厄mploi entre les deux périodes.

\section{CONCLUSIONS}

Les décompositions adoptées dans ce travail permettent $\mathrm{d}$ une part d apprécier 1 ampleur des gains de productivité trouvant leur origine au sein des entreprises (relevant des décisions stratégiques des entreprises) et dautre part de déterminer 1 importante des gains de productivités liées aux réallocations demplois entre entreprises : ces mouvements entre entreprises constituent une force tendant à accroître la productivité siils ont lieu depuis les entreprises les moins productives vers les entreprises les plus productives. À ces deux forces, $\mathrm{s}$ en ajoute une autre liée à 1 effet des créations et disparitions d entreprises industrielles.

\footnotetext{
7 Dans 1 industrie, les taux annuels de création et de destruction atteignent environ $10 \%$. En prenant en compte, la pérennité des créations et des destructions ( $80 \%$ pour les créations et $90 \%$ pour les destructions en moyenne), les taux quinquennaux de création et destruction sont inférieurs à la somme des taux annuels.
} 
Cette dernière composante permet $\mathrm{d}$ appréhender 1 incidence de renouvellement du tissu productif sur la variation de la productivité.

Nous montrons trois résultats concernant 1êconomie marocaine: premièrement, la croissance de la productivité du travail est fortement dépendante de la croissance au sein des entreprises pérennes ; deuxièmement, pour croissance de la productivité globale des facteurs, les entrées jouent un rôle plus important, notamment en incorporant du capital plus performant; enfin, les disparitions d entreprises expliquent la différence de croissance de 1 emploi entre les deux périodes de la décennie 90.

Dans le cas du Maroc, une des pistes à explorer consiste à voir $\square$ à 1 aide de techniques plus explicatives $\square$ dans quelles mesures la libéralisation des années 90 a contribué à 1 augmentation du processus de destruction créatrice, à 1 augmentation de la productivité des entreprises qui ont survécu à cette libéralisation et à en voir 1Ґimpact sur 1๔emploi.

\section{BIBLIOGRAPHIE}

AHN S., 2001, «Firm Dynamics and Productivity Growth: a Review of Micro Evidence from OECD Countries », Economics Department Working Paper, n 297, OCDE.

Baily M., BARTelsman E., J. HaltiwANGer, 2001, «Labor Productivity: Structural Change and Cyclical Dynamics », The Review of Economics and Statistics, vol 83, n 3, pp. 420-433.

Baily M., J. Hulten, J. CAMPBell, 1992, «Productivity dynamics in Manufacturing Plants », Brookings Papers on Economic Activity: microeconomics, pp 187-267.

BALDWIN J., 1995, The Dynamic of Industrial Competition, Cambridge University press.

BARTElSMAN E., M. DOMS, 2000, «Understanding Productivity: Lessons from Longitudinal Microdata », Journal of Economic Litterature, vol 38, $\mathrm{n}^{\circ} 3$.

CAMPBell J., 1997, « Entry, Exit, Embodied Technology and Business Cycle », NBER WP, $\mathrm{n}^{\circ} 5955$.

Caballero R., Hammour M., 1994, «The Cleansing Effect of Recession », American Economic Review, $\mathrm{n}^{\circ}$ 84(5), pp. 1350-1368.

Caballero R., Engel E., Haltiwanger J., 1995, Plant-Level Adjustment and Aggregate Investment Dynamics, Brooking Papers on Economic Activity, n ${ }^{\circ}$ 2, pp. 1-54.

CoOper R., Haltiwanger J., POWer L., 1995, « Machine Replacement and the Business Cycle: Lumps and Bumps », NBER WP, $\mathrm{n}^{\circ} 5260$.

CAVES R., 1998, "Industrial Organization and New Findings on the Turnover and Mobility of Firms », Journal of Economic Literature, nº 36 (4), pp. 1947-1982.

CREPON B., R. Duhautois, 2004, «Ralentissement de la productivité et réallocations demplois : deux régimes de croissance », Economie et Statistique n³67.

DARES, 2003, «Les politiques de 1厄emploi et du marché du travail», Collection Repères $n^{\circ} 373$, La Découverte.

DAVIS S., Haltiwanger J., 1992, «Gross Job Creation, Gross Job Destruction: Microeconomic Evidence and Macroeconomic Implications », NBER macroeconomics annual.

Davis S., Haltiwanger J., 1999, « Gross Job Flows », Handbook of Labour Economics, Vol 3B, Orley and Card editors, pp. 2711-2805.

Disney R., Haskel J., Heden Y., 2000, Restructuring and Productivity Growth in UK Manufacturing, mimeo.

Doms M., DunNE T., 1998, «Capital Adjustment Patterns in Manufacturing Plants », Review of Economic Dynamics, ${ }^{\circ} 1$, pp. 409-429. 
DUHAUTOIS R., 2002, « Les réallocations d emplois en France sont-elles en phase avec le cycle ? », Économie et Statistique, $\mathrm{n}^{\circ}$ 351, pp. 87-103.

DUHAUTOIS R., JAMET S., 2002, «Hétérogénéité des comportements dinvestissement et fluctuations de 1 linvestissement agrégé ", Économie et prévision, n 149, pp. 103-115.

ERICSON R., PAKES A., 1995, « Markov Perfect Industry Dynamics: a Framework for Empirical Analysis », Review of Economic Studies, vol 62, n 1, pp. 53-82.

Foster L, Haltiwanger J. ET KriZAN C., 2000, «Aggregate Productivity Growth: Lessons from Microeconomic Evidence », document présenté au NBER.

GRILICHES Z., REGEV H., 1995, « Productivity and Firm Turnover in Israeli Industry: 1979-1988 », Journal of Econometrics, $\mathrm{n}^{\circ} 65$, pp. 175-203.

HaltiWAnger J., 1997, « Measuring and Anlysing Aggregate Fluctuations: the Importance of Building from Microeconomic Evidence », Federal Reserve Bank of St-Louis review, may/june, pp. 55-77.

JOVANOVIC B., 1982, « Selection and the Evolution of Industry », Econometrica, vol 50, n 3, pp. 649-670.

MAC GUCKIN, STIROH, 1999, «Understanding Productivity Growth: Entry, Survival and the Competitive Process ", the conference board.

VAN DER VIEL, H.P., 1999, «Firm Turnover in Dutch Business Services », document de travail n 159 , Centraal Planbureau.

PAKES A., ERICSON R., 1998, «Empirical Implications of Alternative Models of Firms Dynamics », The journal of Economic Theory, vol 79, pp. 1-45. 


\section{ANNEXE}

Tableau 1

Décomposition de la croissance de la productivité selon FHK et GR

\section{A - Productivité du travail}

Méthode FHK

\begin{tabular}{|c|c|c|c|c|c|c|c|}
\hline & $\Delta P_{t}(\%)$ & Intra & Inter & Covariation & $\begin{array}{c}\text { Entrée } \\
\text { nette }\end{array}$ & $\begin{array}{c}\text { Entrées } \\
\text { brutes }\end{array}$ & $\begin{array}{c}\text { Sorties } \\
\text { brutes }\end{array}$ \\
\hline $1990-1995$ & 5,6 & 10,3 & 0,2 & $-6,5$ & 1,6 & $-7,6$ & 9,2 \\
\hline $1995-2000$ & 2,8 & 7,1 & 4,5 & $-9,2$ & 0,5 & $-8,2$ & 8,7 \\
\hline
\end{tabular}

Lecture : la productivité du travail a augmenté de 5,8\% entre 1990 et 1995 . La contribution intra est de 10,8 points, la contribution inter de 0,2 , la contribution de la covariation de $-6,5$ et la contribution des entrées moins les sorties de 1,6, la somme de ces composantes étant égale au taux de croissance.

Méthode GR

\begin{tabular}{|c|c|c|c|c|c|c|c|}
\hline & $\Delta P_{t}(\%)$ & Intra & Inter & Covariation & $\begin{array}{c}\text { Entrée } \\
\text { nette }\end{array}$ & Entrées & Sorties \\
\hline $1990-1995$ & 5,6 & 7,1 & $-3,0$ & & 1,5 & $-8,1$ & 9,6 \\
\hline $1995-2000$ & 2,8 & 2,4 & $-0,3$ & & 0,7 & $-8,4$ & 9,1 \\
\hline
\end{tabular}

\section{B - Productivité globale des facteurs}

Méthode FHK

\begin{tabular}{|c|c|c|c|c|c|c|c|}
\hline & $\Delta P_{t}(\%)$ & Intra & Inter & Covariation & $\begin{array}{c}\text { Entrée } \\
\text { nette }\end{array}$ & Entrées & Sorties \\
\hline $1990-1995$ & $-1,6$ & $-0,8$ & $-0,7$ & $-1,9$ & 1,8 & $-5,0$ & 6,8 \\
\hline $1995-2000$ & 1,5 & 0,9 & 3,2 & $-4,3$ & 1,7 & $-3,3$ & 5,0 \\
\hline
\end{tabular}

Méthode GR

\begin{tabular}{|c|c|c|c|c|c|c|c|}
\hline & $\Delta P_{t}(\%)$ & Intra & Inter & Covariation & $\begin{array}{c}\text { Entrée } \\
\text { nette }\end{array}$ & Entrées & Sorties \\
\hline $1990-1995$ & $-1,6$ & $-1,7$ & $-1,7$ & - & 1,8 & $-4,8$ & 6,6 \\
\hline $1995-2000$ & 1,5 & $-1,2$ & 0,9 & - & 1,8 & $-3,5$ & 5,3 \\
\hline
\end{tabular}


Tableau 2

Décomposition de la croissance de la productivité par secteurs selon FHK

\section{A - Productivité du travail}

Secteur Agro-alimentaire

\begin{tabular}{|c|c|c|c|c|c|c|c|}
\hline & $\Delta P_{t}(\%)$ & Intra & Inter & Covariation & $\begin{array}{c}\text { Entrée } \\
\text { nette }\end{array}$ & Entrée & Sortie \\
\hline $1990-1995$ & 5,6 & 18,0 & 3,7 & $-11,0$ & $-5,1$ & $-7,8$ & 2,8 \\
\hline $1995-2000$ & 20,0 & 21,9 & 13,6 & $-18,5$ & 3,0 & $-4,8$ & 7,8 \\
\hline
\end{tabular}

Secteur Chimie et Parachimie

\begin{tabular}{|c|c|c|c|c|c|c|c|}
\hline & $\Delta P_{t}(\%)$ & Intra & Inter & Covariation & $\begin{array}{c}\text { Entrée } \\
\text { nette }\end{array}$ & Entrée & Sortie \\
\hline $1990-1995$ & 9,2 & 10,0 & 1,0 & $-6,1$ & 4,2 & $-5,1$ & 9,3 \\
\hline $1995-2000$ & 17,8 & 6,7 & 11,1 & $-7,0$ & 7,0 & $-4,5$ & 11,5 \\
\hline
\end{tabular}

Secteur Mécanique et Métallurgie

\begin{tabular}{|c|c|c|c|c|c|c|c|}
\hline & $\Delta P_{t}(\%)$ & Intra & Inter & Covariation & $\begin{array}{c}\text { Entrée } \\
\text { nette }\end{array}$ & Entrée & Sortie \\
\hline $1990-1995$ & $-10,8$ & $-7,7$ & 5,7 & $-4,6$ & $-4,3$ & $-10,2$ & 5,9 \\
\hline $1995-2000$ & 4,5 & 9,6 & 2,8 & $-7,6$ & $-0,2$ & $-6,1$ & 5,8 \\
\hline
\end{tabular}

Secteur Textile et Cuir

\begin{tabular}{|c|c|c|c|c|c|c|c|}
\hline & $\Delta P_{t}(\%)$ & Intra & Inter & Covariation & $\begin{array}{c}\text { Entrée } \\
\text { nette }\end{array}$ & Entrée & Sortie \\
\hline $1990-1995$ & 8,4 & 12,0 & $-1,7$ & $-5,8$ & 3,9 & $-2,7$ & 6,6 \\
\hline $1995-2000$ & $-2,9$ & 1,5 & 2,2 & $-7,0$ & 0,4 & $-4,8$ & 5,2 \\
\hline
\end{tabular}




\section{B - Productivité globale des facteurs}

Secteur Agro-alimentaire

\begin{tabular}{|c|c|c|c|c|c|c|c|}
\hline & $\Delta P_{t}(\%)$ & Intra & Inter & Covariation & $\begin{array}{c}\text { Entrée } \\
\text { nette }\end{array}$ & Entrée & Sortie \\
\hline $1990-1995$ & $-6,7$ & 0,7 & 1,6 & $-6,0$ & $-3,0$ & $-6,3$ & 3,3 \\
\hline $1995-2000$ & 14,2 & 14,2 & 9,9 & $-11,8$ & 1,8 & $-4,4$ & 6,2 \\
\hline
\end{tabular}

Secteur Chimie et Parachimie

\begin{tabular}{|c|c|c|c|c|c|c|c|}
\hline & $\Delta P_{t}(\%)$ & Intra & Inter & Covariation & $\begin{array}{c}\text { Entrée } \\
\text { nette }\end{array}$ & Entrée & Sortie \\
\hline $1990-1995$ & $-4,6$ & $-3,3$ & 0,1 & $-2,8$ & 1,4 & $-5,1$ & 6,5 \\
\hline $1995-2000$ & 6,7 & 0,8 & 6,3 & $-3,6$ & 3,2 & $-2,8$ & 6,0 \\
\hline
\end{tabular}

Secteur Mécanique et Métallurgie

\begin{tabular}{|c|c|c|c|c|c|c|c|}
\hline & $\Delta P_{t}(\%)$ & Intra & Inter & Covariation & $\begin{array}{c}\text { Entrée } \\
\text { nette }\end{array}$ & Entrée & Sortie \\
\hline $1990-1995$ & $-17,6$ & $-15,0$ & 2,8 & $-2,7$ & $-2,7$ & $-8,3$ & 5,6 \\
\hline $1995-2000$ & 6,0 & 5,3 & 1,3 & $-1,6$ & 1,0 & $-3,0$ & 4,0 \\
\hline
\end{tabular}

Secteur Textile et Cuir

\begin{tabular}{|c|c|c|c|c|c|c|c|}
\hline & $\Delta P_{t}(\%)$ & Intra & Inter & Covariation & $\begin{array}{c}\text { Entrée } \\
\text { nette }\end{array}$ & Entrée & Sortie \\
\hline $1990-1995$ & 5,8 & 3,3 & $-2,1$ & $-0,1$ & 4,7 & 0,2 & 4,5 \\
\hline $1995-2000$ & $-1,8$ & $-4,8$ & 2,0 & $-2,1$ & 3,1 & 0,4 & 2,7 \\
\hline
\end{tabular}

Tableau 3

Flux bruts d emplois (ensemble des entreprises)

\begin{tabular}{|c|c|c|c|c|}
\hline & $\begin{array}{c}\text { Taux de croissance } \\
\text { nette }\end{array}$ & Taux de création & Taux de destruction & Taux de réallocation \\
\hline $1990-1995$ & 6,9 & 34,1 & 27,2 & 61,3 \\
\hline $1995-2000$ & 2,1 & 35,0 & 32,9 & 67,9 \\
\hline
\end{tabular}




\section{Tableau 4}

Taux de création et de destruction dans chaque classe

\begin{tabular}{|c|c|c|c|c|c|}
\hline & Créations & $\begin{array}{c}\text { Pérennes } \\
\text { créatrice }\end{array}$ & Stable & $\begin{array}{c}\text { Pérennes } \\
\text { destructrice }\end{array}$ & Disparitions \\
\hline & $(1 \mathrm{a})$ & $(2 \mathrm{a})$ & stable & $(3 \mathrm{a})$ & $(4 \mathrm{a})$ \\
\hline $1990-1995$ & 17,3 & 16,8 & 0 & $-10,5$ & $-16,7$ \\
\hline $1995-2000$ & 14,0 & 21,0 & 0 & $-10,6$ & $-22,2$ \\
\hline
\end{tabular}

Tableau 5

Distribution de la taille des entreprises

\begin{tabular}{|c|c|c|c|c|c|}
\hline & $1^{\text {er }}$ décile & $1^{\text {er }}$ quartile & médiane & $3^{\text {e }}$ quartile & $9^{\text {e }}$ décile \\
\hline 1990 & 5 & 9 & 20 & 62 & 175 \\
\hline 1995 & 5 & 9 & 25 & 75 & 200 \\
\hline 2000 & 4 & 7 & 17 & 56 & 169 \\
\hline
\end{tabular}




\section{DERNIERS NUMEROS PARUS :}

téléchargeables à partir du site

http://www.cee-recherche.fr

$\mathbf{N}^{\circ} 53$ PME et industrialisation: Que sont devenues les PME du "miracle choletais » (19452004)?

BRUNO COURAULT

décembre 2005

$\mathbf{N}^{\circ} 52$ La révélation des préférences éthiques pour la redistribution: comparaison de la portée de différentes méthodes empiriques

Christine Le Clainche

décembre 2005

$\mathbf{N}^{\circ} \mathbf{5 1}$ La qualité de l Lemploi en France : tendance et cycle

FLORENT FREMIGACCI, YANNICK LIHORTY

novembre 2005

$\mathbf{N}^{\circ} \mathbf{5 0}$ Job Board Toolkits: Internet Matchmaking and the Transformation of Help-Wanted Ads

Emmanuelle Marchal, KeVin Mellet, Geraldine Rieucau

novembre 2005

$N^{\circ} 49$ Economic Regionalization and Industrial Relations

ISABEL DA COSTA

novembre 2005

$N^{\circ} 48$ Pluralité des marchés du travail et qualité des intermédiaires

Marie-Christine Bureau, EMmanuelle Marchal

novembre 2005

$\mathbf{N}^{\circ} 47$ Le prix du marché. Enquêtes de rémunération et mise en forme du marché du travail dans lindustrie financière

OLIVIER GODECHOT

septembre 2005

$\mathbf{N}^{\circ} 46$ Défauts de coopération et chômage : une théorie institutionnaliste FRANÇOIS EYMARD-DUVERNAY

septembre 2005

$N^{\circ} 45$ Emploi des mères et politique familiale : doit-on s inspirer du « modèle suédois »?

Celine Marc, Helene ZaJdela

septembre 2005

$\boldsymbol{N}^{\circ} 44$ Hold-up en finance. Les conditions de possibilité des bonus élevés dans l lindustrie financière OLIVIER GODECHOT

septembre 2005

$N^{\circ} 43$ Les conditions du travail en équipe. Post-enquête "Conditions et organisation du travail dans les établissements de santé » MIHAÏ DINU GHEORGHIU, FREDERIC MOATTY juillet 2005 